\title{
Le guano, fertilisant organique naturel alternatif au fumier, testé sur la production de la laitue verte (lactuca sativa $L$.) dans l'oasis de Balla (département de Gouré), Zinder, Niger
}

\author{
Moustapha Maman MOUNIROU ${ }^{1,4^{*}}$, Karimou Ambouta HAROUNA², \\ Adamou Didier TIDJANI ${ }^{1}$ et Malam Hassane MAIGARI ${ }^{3}$ \\ ${ }^{1}$ Université Abdou Moumouni de Niamey, Faculté d'Agronomie, BP 10960, Niger. \\ ${ }^{2}$ Université Polytechnique de Bobo-Dioulasso, 01 BP 1091 Bobo-Dioulasso 01. \\ ${ }^{3}$ Université de Diffa, Faculté des Sciences Agronomiques, BP : 78 Diffa-Niger. \\ ${ }^{4}$ Université d'Ankara, Faculté d'Agronomie, Département des Sciences du Sol et Nutrition des Plantes, TR- \\ 06110 Ankara-Turquie. \\ "Auteur correspondant ; E-mail : mounirhalifamoustapha@gmail.com; Tel: +227 98171736
}

\section{REMERCIEMENTS}

Nous remercions infiniment le projet PIC-REC 2 d'avoir financé cette recherche.

\section{RESUME}

Au Niger Est, la faible disponibilité sur les marchés ruraux, la cherté et le faible pouvoir d'achat des paysans limitent l'application des engrais minéraux. L'objectif de cette étude est l'évaluation de l'effet du guano sur la production de la laitue. Le dispositif expérimental est un bloc complet randomisé avec dix-sept traitements issus de la combinaison entre 4 doses de guano, 2 doses de fumier et 2 doses d'engrais en trois (3) répétitions. Les paramètres étudiés portent sur les diamètres de pomme, la hauteur des parties aériennes, le développement foliaire et le rendement. Les résultats obtenus révèlent que le guano a un effet significatif sur la production de laitue. A la dose optimale 3,5 t/ha, le guano a permis d'augmenter la production de la laitue de 833 et $628 \%$ par rapport au témoin et aux parcelles ayant reçues uniquement la fumure minérale. La combinaison guano et fumure organique a permis d'accroitre le rendement par rapport aux parcelles fertilisées avec le guano seulement, tandis que l'apport de l'engrais minéral a un effet dépressif sur le rendement. Pour assurer une bonne production de laitue dans la cuvette de Balla, il serait préférable d'utiliser la combinaison guano (3,5 t/ha) avec du fumier. La préservation de la population de chauves-souris permettrait d'assurer la durabilité de la filière guano.

(C) 2020 International Formulae Group. All rights reserved.

Mots clés : Fertilisation, guano, laitue, cuvette oasienne, Gouré.

\section{Guano, a natural organic fertilizer alternative to manure, tested on the production of green lettuce (lactuca sativa L.) in oasis of Balla (department of Gouré), Zinder, Niger}

\begin{abstract}
In eastern Niger, low availability in local markets, high prices and low purchasing power of farmers limit the application of mineral fertilizers. The objective of this study is to evaluate the effect of guano on lettuce production. The experimental design is a randomized complete block with seventeen treatments resulting from the combination of four doses of guano, two doses of manure and two doses of fertilizer in three (3) replicates.
\end{abstract}


The parameters studied for this experiment are: diameter of head, cabbage height of aerial parts, leaf development and yield. The results show that guano had a significant effect on lettuce production. At the optimum rate of 3,5 t/ha, guano increased lettuce production by 833 and $628 \%$ compared with the control and the plots receiving only the mineral fertilizer respectively. The combination of guano and organic fertilizer increased the yield compared to the plots fertilized with guano only, while the mineral fertilizer input had a depressive effect on yield. To ensure a good lettuce production in the Balla lowland, it would be better to use the guano combination (3,5 t/ha) with manure. Preserving the bat population would help ensure the sustainability of the guano industry.

(C) 2020 International Formulae Group. All rights reserved.

Keywords: Fertilizer, guano, lettuce, Oasis bassin, Gouré.

\section{INTRODUCTION}

$\mathrm{Au}$ Niger, l'agriculture constitue la principale activité avec plus de $80 \%$ de la population active qui la pratique pour subvenir à leurs besoins alimentaires et économiques. Elle contribue à plus de $40 \%$ du produit intérieur brut (FAO, 2006; Ndiaye et al., 2013). Depuis le début des années 1970, cette agriculture a commencé à payer de façon croissante le poids des effets combinés de la pression démographique et de la variabilité climatique. Dans les systèmes de production ruraux, les conséquences les plus perceptibles sont la baisse de la fertilité des sols et de la production agricole (Guengant, 2003 ; Habou et al., 2016). Cette situation accentue ainsi le déséquilibre entre la production nationale et les besoins en denrées alimentaires de la population. Face à cette situation, la promotion de la culture irriguée peu dépendante des caprices climatiques représente une bonne alternative d'amélioration de la production agricole.

Au Niger Est, plus précisément au département de Gouré, l'utilisation comme fertilisant de fond et d'appoint du guano des chauves-souris commence à se développer. En effet, ce type d'amendement a prouvé son efficacité dans de nombreux systèmes de production et peut être considéré comme une alternative aux engrais (Mentler et al., 2002 ; Karen, 2003). Dans le contexte du système oasien du Manga, la découverte et l'utilisation de ce fertilisant constitue une bonne opportunité pour pallier au déficit d'engrais organique et minéral d'une part, et aux faibles productions d'autre part. Dans les revenus des ménages du Manga, les retombées issues de l'exploitation des cuvettes oasiennes contribuent à plus de $50 \%$ contre $15 \%$ pour les cultures pluviales (Malam Boukar et al., 2016). Dans ce contexte, promouvoir l'utilisation du guano de Magarawa contribuera certainement à améliorer la production maraichère. Les performances agronomiques du guano se traduisent par l'augmentation de la vitesse de croissance des plantes et l'amélioration de l'équilibre minéral du sol. Il convient tant aux cultures maraichères qu'aux cultures pluviales $\mathrm{du}$ fait de sa composition et de ses caractéristiques. Le guano utilisé comme engrais organique est riche en $\mathrm{P}_{2} \mathrm{O}_{5}$ (peroxyde de phosphore) et en Azote. Sa richesse en nutriments (azote, phosphore et oligoélements) lui confère un effet "coup de fouet" remarquable, parmi les plus rapides dans les engrais naturels (Sridhar et al., 2006; Mulambuila et al. 2015). Il peut être utilisé à la préparation du sol, avant semis, repiquage ou plantation en fonction du sol et du besoin des cultures, tout en arrosant abondamment le sol (Karimou, 2013).

Pour évaluer l'efficacité du guano de Magarawa, la cuvette à eau profonde de Balla a été choisi du fait de l'expérience des exploitants dans l'utilisation dudit produit en maraichage. Cependant, aucune étude n'indique si les dosages sont adéquats ou pas, d'où cette étude. Elle a pour objectif de comparer l'efficacité de ce type de guano avec les fertilisants couramment utilisés dans la production de la laitue verte dans le système oasien du Manga. Sur le plan spécifique, ce travail permettra d'analyser les différents maillons de la collecte à l'utilisation du guano en maraichage, de caractériser la composition chimique du guano et de mesurer l'effet du guano sur le rendement de la laitue verte dans 
un contexte de comparaison avec les autres fertilisants.

\section{MATERIEL ET METHODES Sites d'étude}

Le guano utilisé dans ce travail a été prélevé au niveau des collines du village de Magarawa (Sud-ouest de la ville de Gouré), les essais agronomiques se sont déroulés dans la cuvette de Balla (Sud-est). Les sols dans cette cuvette sont de texture limoneuse et présentent comme principales contraintes la salinité et la faible teneur en matière organique (Figure 1).

\section{Echantillon d'enquête}

Les producteurs maraichers travaillant dans la cuvette de Balla et les collecteurs du guano du village de Magarawa ont servi de support aux enquêtes autour de l'objectif de l'étude. Quinz (15) collecteurs du guano basés à Magarawa, sur les 30 exploitants de cette ressource ont été enquetés, soit un taux d'échantillonnage de $50 \%$. Le guano prélevé est commercialisé par une seule personne dans le département de Gouré. Cette personne a permis d'obtenir les informations sur la filière. $\mathrm{Au}$ niveau de Balla (Site de l'essai agronomique), il a été recensé 93 producteurs maraichers pour l'ensemble de la population. Sur la base du nombre total de ces maraichers, un taux de sondage de $20 \%$ a été appliqué, soit 19 producteurs.

\section{Matériel végétal}

Le matériel végétal utilisé pour tester l'efficacité du guano en culture maraîchère était Lactuca sativa $\mathrm{L}$. de la variété Maya. C'est une variété à grosse pomme ferme, résistante à la sécheresse et à pollinisation ouverte. Elle est adaptée aux conditions de températures élevées et résistante à la montaison. Le poids moyen à la récolte varie de 300 à $800 \mathrm{~g}$ par pomme selon la saison de culture. Cette variété Maya a été selectionnée au Niger par l'ICRISAT (Karimou, 2013; RECA, 2015).

\section{Guano, fertilisant naturel}

Le guano est le nom donné aux excréments des chauves-souris. Il est récolté dans diverses grottes habitées par des chauvessouris de la colline de Magarawa. Au moment de l'étude, le guano (Photo 1) était accumulé sur plusieurs mètres d'épaisseur.

\section{Dispositif expérimental}

Le dispositif expérimental utilisé était un bloc complètement randomisé avec dix-sept traitements répétés trois fois. Chaque bloc est composé de dix-sept parcelles de $1 \mathrm{~m}^{2}$ espacées entre elles de $0,5 \mathrm{~m}$. Les blocs quant à eux sont distants de $1 \mathrm{~m}$. Le dispositif entier s'étend sur une surface de $126,5 \mathrm{~m}^{2}(23 \mathrm{~m} \times 5,5 \mathrm{~m})$ présentant un sol de texture limoneuse. L'eau d'irrigation utilisée provient d'un même puits. Pour les analyses des paramètres étudiés, différents traitements sont renommés afin de faciliter la lecture. Les traitements se présentent comme suit :

1.- Témoin sans fertilisant « $\mathrm{T} 0 »$;

2.- Guano (dose moyenne) « $\mathrm{G} 2 »(6,5 \mathrm{t} / \mathrm{ha})$;

3.- Guano (dose faible) « G1» $(3,5 \mathrm{t} / \mathrm{ha})$;

4.- Guano (dose forte) « $\mathrm{G} 3 »(10 \mathrm{t} / \mathrm{ha})$;

5.- NPK + Urée (dose recommandée) «FMV » (200 kg/ha NPK 15-15-15 + 50 kg/ha Urée) ; 6.- Fumier dose recommandée «FOV » $(25$ t/ha);

7.- NPK + Urée dose recommandée + Fumier dose vulgarisée «FMV + FOV »;

8.- Guano (dose moyenne)+NPK + Urée dose recommandée + Fumier dose recommandée « $\mathrm{G} 2+\mathrm{FMV}+\mathrm{FOV} »$;

9.- Guano (dose faible)+ NPK + Urée dose recommandée + Fumier dose recommandée « G1+FMV+FOV »;

10.- Guano (dose faible)+ NPK + Urée dose recommandée + Fumier dose recommandée « G3+FMV+FOV »;

12.- Guano (dose moyenne)+ NPK + Urée dose recommandée « G2+FMV »;

12.- Guano (dose faible)+ NPK + Urée dose recommandée « G1+FMV »;

13.- Guano (dose forte)+ NPK + Urée dose recommandée « G3+FMV»;

14.- Guano (dose moyenne)+ Fumier dose recommandée « $\mathrm{G} 2+\mathrm{FOV}$ »;

15.- Guano (dose faible)+ Fumier dose recommandée « $\mathrm{G} 1+\mathrm{FOV} »$;

16.- Guano (dose forte)+ Fumier dose 
recommandée « G3+FOV »;

17.- Pratique paysanne « $\mathrm{PP} »(10 \mathrm{t} / \mathrm{ha}$ fumier $+300 \mathrm{~kg} / \mathrm{ha} \mathrm{NPK}+150 \mathrm{~kg} / \mathrm{ha}$ Urée + G2).

\section{Echantillonnage du sol et analyse physico- chimique du sol et des amendements organiques}

$\mathrm{Au}$ sein de la parcelle expérimentale située dans le secteur présentant de bonnes aptitudes aux cultures maraichères de la cuvette de Balla, un échantillon composite de sol prélevé à $0-20 \mathrm{~cm}$ a été constitué sur la base d'un prélèvement aléatoire. L'analyse granulométrique de l'échantillon de sol et les analyses physico-chimiques $(\mathrm{pH}$, carbone organique total, azote total, phosphore total, bases échangeables et capacité d'échange cationique) ont été réalisées suivant les indications du guide d'analyse des sols de la FAO.

Le pH (eau) des sols et des amendements organiques ont été mesurés avec un pH-mètre à électrodes en verre (Tac Ussel). La solution utilisée pour la lecture a été préparée dans un rapport terre/eau 1/2.5. Le carbone organique a été dosé par la méthode de Walkley-Black (Walkley et Black, 1934). Pour le dosage de l'Azote, la méthode Kjeldahl a été utilisée, le phosphore $(\mathrm{P})$ total par la méthode de Bray 1, les bases échangeables et la capacité d'échange cationique (CEC) par la méthode Argent Thiourée (Dickman et Bray, 1940 ; Hillebrand et al. 1953 ; Jackson, 1958 ; Pratt, 1965). La texture du sol a été déterminée par la méthode de Bouyoucos (Bouyoucos, 1951).

\section{Mesures et observations agronomiques}

Pour évaluer l'effet du guano sur la production de la laitue verte, il est mesuré la surface foliaire, le diamètre de la pomme, la hauteur des parties aériennes, le poids frais et sec de la laitue. Pour mesurer la surface foliaire, la longueur a été multipliée par la largeur des feuilles tout en sachant que la feuille n'est rectangulaire. Dans ces conditions, l'erreur est la même pour toutes les mesures.

\section{Analyses statistiques des données}

Le tableur Excel a été utilisé pour le traitement des données des enquêtes et la constitution des bases de données de toutes les mesures effectuées. Le logiciel GenStat Discovery Edition 4.1 a été utilisé pour les analyses statistiques des données. Les résultats relatifs à l'étude du rendement de laitue ont été soumis à une analyse de variance (ANOVA). Pour la séparation des moyennes, elle a été réalisée avec le test de la Plus Petite Différence Significative (PPDS) au seuil de 5\%.

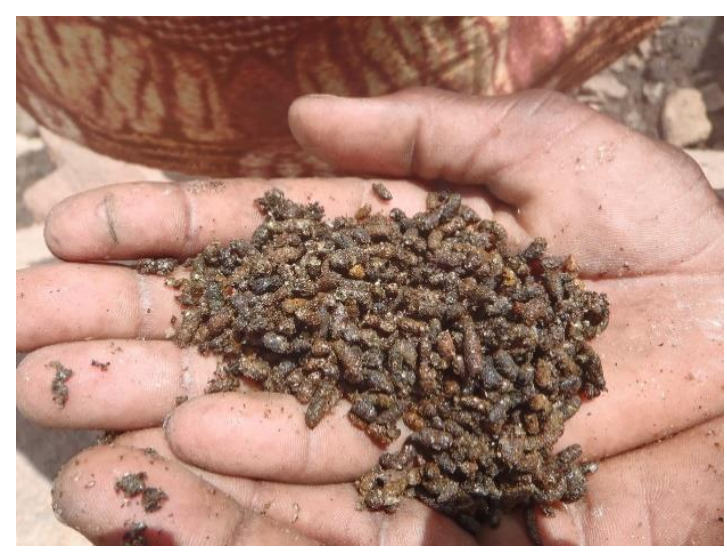




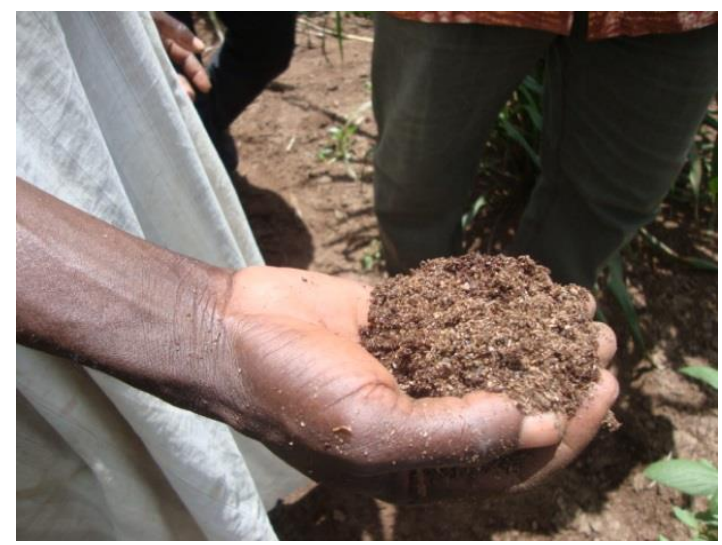

Photo 1 : Guano de chauve-souris de Magarawa.

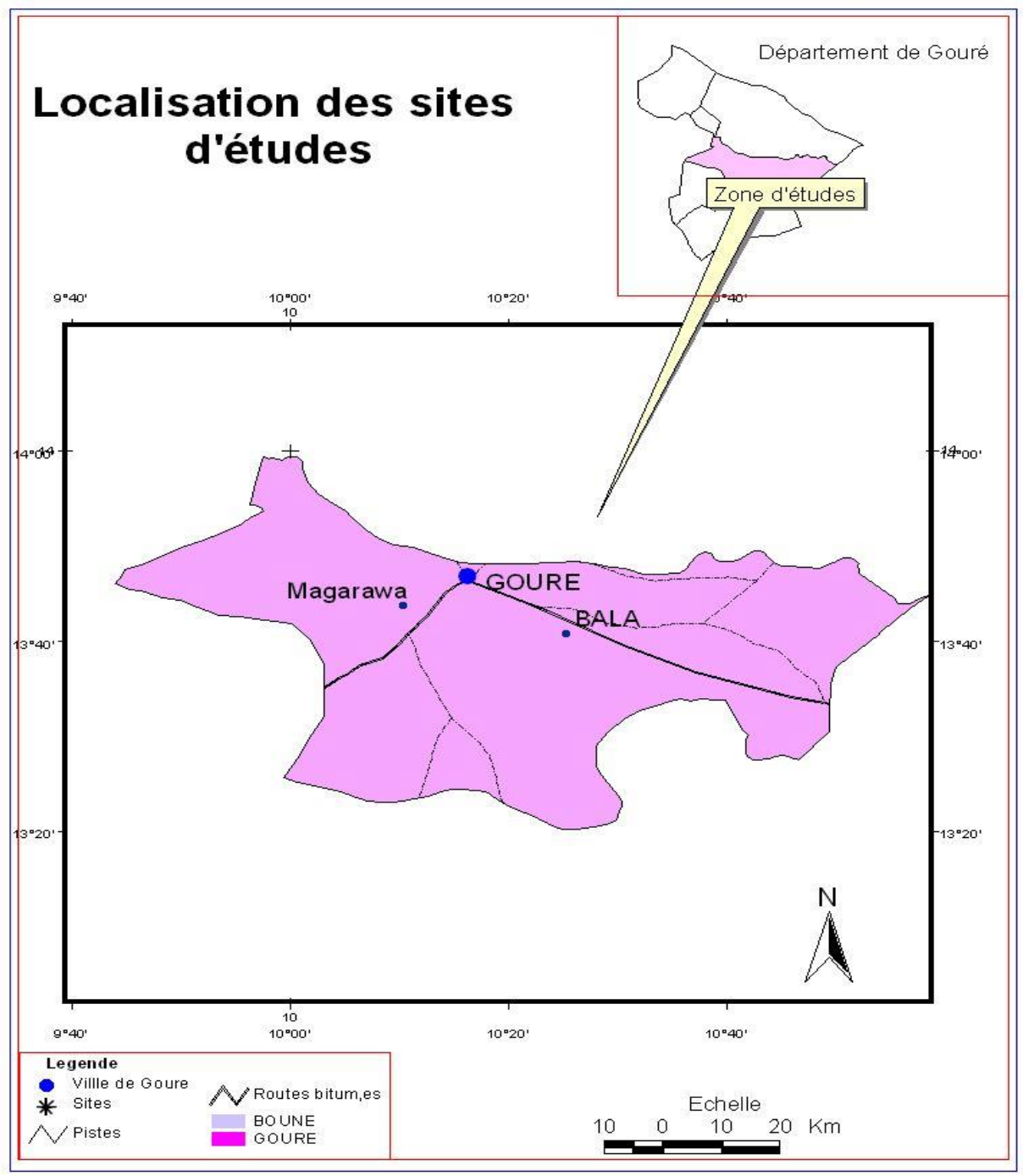

Figure 1 : Carte de localisation du département de Gouré et des villages d'étude. 


\section{RESULTATS}

\section{Exploitation du guano \\ Caractéristiques du site de production du guano}

Le site de production du guano situé dans la colline de Magarawa est un chaos granitique truffé de grottes de tailles variables. Ce secteur est dénommé en Kanouri « Bourja » (refuge de l'hyène) est située dans le Mounio $\left(10^{\circ} 08^{\prime} 00,7^{\prime}\right.$ 'E, $13^{\circ} 53^{\prime} 42,9^{\prime}$ 'N) à $20 \mathrm{~km}$ au Sud-Ouest du département de Gouré. Elle est entourée par des champs de mil, de sorgho, de l'oseille et un pâturage. Du fait de la présence du guano épandu par le ruissellement au piémont de la colline, la production agricole est estimée y être la plus élevée. Pour le mil, la production en grains est de 2,35 tonnes/ha tandis que la moyenne de production dans la zone est de $500 \mathrm{~kg} / \mathrm{ha}$. Les chauves-souris productrices du guano se fixent sur les parois des blocs de granite de la colline non exposées au soleil. Cet habitat des chauves-souris se caractérise par la présence de plafonds rocheux, de voûtes, des aspérités et des parois obscures. Elles ne sont visibles que pendant la saison des pluies et disparaissent en saison sèche. Pour ne pas perturber leur vie, le guano n'est prélevé qu'en saison sèche.

\section{Collecte du guano et difficultés rencontrées}

Il existe à Magarawa 30 collecteurs de guano, tous de sexe masculin âgés de 12 à 30 ans, dont $48 \%$ sont des célibataires et $52 \%$ des mariés. L'activité de collecte du guano à Magarawa a débuté en 2002. Plusieurs outils sont utilisés pour la récolte du guano. Il s'agit de sacs en polyéthylène de $100 \mathrm{~cm}$ de long et de $60 \mathrm{~cm}$ de largeur, de lampes torche pour illuminer les grottes, de pelles et de tasses pour collecter le guano et d'un couteau pour se défendre en cas d'attaque de serpent. Les étapes de la collecte sont : i) escalader la colline en prenant toutes les précautions pour ne pas tomber ou se faire piquer par les insectes ; ii) pénétrer dans les grottes disposant d'ouvertures pouvant permettre à un être humain de s'y glisser (25 cm à $1 \mathrm{~m}$ de large) ; iii) accéder au guano et se servir selon ses capacités ; iv) faire sortir les sacs remplis un à un et les acheminer au village sur des charrettes.

Les plus jeunes collecteurs dont l'âge varie de 12 à 15 ans soit $47 \%$ des enquêtés collectent chacun moins de 100 sacs et les autres plus âgés extraient 100 à 300 sacs par an. La saison sèche chaude (mars-mai) constitue la période la plus difficile pour récolter le guano du fait des températures excessives dans la colline, contrairement à la saison sèche et froide (novembre-février).

Les difficultés rencontrées dans la collecte du guano sont la prévalence de risque de chute et de blessure des collecteurs, les morsures de serpents, les déchirures des sacs, la pénibilité de sortir les sacs remplis de la grotte, les risques d'infections pulmonaires du fait de la forte odeur de l'ammoniac. Pour améliorer les conditions de la récolte du guano, les collecteurs de ce village proposent : i) Une prise de précaution en vue de réduire les risques liés à l'exploitation; ii) l'utilisation des habits de protection et des outils de collecte adéquats, iii) la création d'une coopérative pour gérer durablement l'exploitation de cette ressource naturelle.

\section{Commercialisation du guano}

La commercialisation du guano est une activité peu pratiquée dans l'ensemble de la zone de Gouré. Deux commerçants seulement ont été identifiés. Le premier dont l'activité reste très locale, vient du village voisin d'Issufuri, distant de moins de $5 \mathrm{~km}$ de Magarawa et le second vient de Guidimouni, une localité située à $80 \mathrm{~km}$ de la colline. Ce dernier âgé de 50 ans est le seul qui approvisionne la zone de Gouré en guano et une partie de Mirriah. Selon les enquêtés, le circuit de commercialisation du guano est organisé suivant deux axes : des grossistes aux maraichers pour le premier et par l'intermédiaire des commerçants détaillants pour le second.

Pour la promotion de l'utilisation du guano dans les cultures maraichères, l'actuel grossiste donnait gratuitement le guano aux maraichers. Par la suite, ayant constaté l'engouement et la demande croissante, la commercialisation avait commencé dans la cuvette de Guidimouni (site pionnier de l'utilisation du guano). Une année plus tard à Guidiguir et de plus en plus à Balla, Ririya, 
Soubdou et Hamdara. Le guano est aussi vendu à Kilakina, Sissia et Gouchi (Figure 2). Tous ces sites maraichers se trouvant dans le système oasien ou dans la Korama. Les maraichers de Guru au Nigeria ont commencé aussi à s'y intéresser depuis 2009.

Cet engouement progressif pour le Guano a fait croître le prix d'achat et de vente qui ont pratiquement quadruplé entre 2008 et 2012. Pour la même période, le bénéfice généré par sac a été multiplié par plus de 7 (Tableau 1). Cette augmentation des prix s'explique par le rôle que joue le guano dans l'amélioration de la production maraichère dans les oasis du Manga.

Le prix du guano peut être variable suivant l'offre et la demande qui est fortement influencée par les périodes des cultures maraichères (Décembre à Mars). Généralement, il est plus élevé en début de saison sèche et froide, car les activités maraichères sont plus intenses à cette période. Un commerçant détaillant du village de Hamdara affirme qu'il a vendu en 2011 un sac à $4000 \mathrm{fcfa}$ pour un prix d'achat de $3000 \mathrm{fcfa}$ chez le grossiste. La période active de vente du guano va d'octobre à mars, moment propice pour les cultures maraîchères. Dans cette zone d'étude, personne n'achète le guano pour les besoins de culture pluviale. Tout de même, les exploitants de Mirriah utilisent parfois le guano pour la culture d'arachide. Les contraintes principales liées à l'exploitation du guano dans cette zone sont l'insuffisance de moyens adéquats de transport du guano de la colline à la route nationale $\mathrm{N}^{\circ} 1$, la méconnaissance du stock du guano disponible à Magarawa et l'éthologie de la chauve-souris.

\section{Expérimentation agronomique \\ Caractéristiques physico-chimiques du sol, support de l'expérimentation}

Les résultats des analyses physicochimiques du sol de la parcelle expérimentale (Tableau 2) montrent que, la texture est limoneuse, le $\mathrm{pH}_{\text {eau }}$ est extrêmement alcalin, la teneur en matière organique et en azote est faible et le rapport $\mathrm{C} / \mathrm{N}$ indique que la matière organique est peu minéralisée d'où une mauvaise humification de celle-ci.

\section{Caractéristiques physico-chimiques du Guano de Magarawa et du fumier de Balla}

Le Tableau 3 présente les caractéristiques physico-chimiques du guano de Magarawa et du fumier de Balla. Les caractéristiques chimiques du guano sont supérieures à celles du fumier (Tableau 3). L'interprétation des résultats d'analyse montre tant pour le fumier que pour le guano que le $\mathrm{pH}$ est alcalin, la conductivité électrique est non saline, et la teneur en matière organique très élevée. Cependant, le fait que les teneurs en $\mathrm{N}$, $\mathrm{P}, \mathrm{K}, \mathrm{C}$ du guano soient nettement supérieures à celles du fumier fait du guano de Magarawa une substance à forte valeur fertilisante.

\section{Effet du guano sur la croissance végétative de laitue}

Dans le Tableau 4 sont consignés les résultats de l'effet des traitements sur la croissance végétative de la laitue. Les traitements ont un effet significatif sur tous les paramètres de la croissance végétative de la laitue verte. En effet l'analyse statistique montre une différence très significative $(\mathrm{F}$ prob : 0,008) entre les combinaisons G3+FOV, G1, G2+FOV, G2+FOV+FMV et PP par rapport au témoin $\mathrm{T} 0$ pour la surface foliaire. Le traitement G1 (faible dose) permet d'obtenir une surface foliaire statistiquement identique à ceux des traitements $\mathrm{G} 2+\mathrm{FOV}+\mathrm{FMV}$ et G3+FOV. La tendance montre également que, pour chacune des doses du guano G1, G2 et G3, un apport de la fumure minérale n'a aucun effet sur l'évolution de la surface foliaire. Par contre l'apport du fumier au guano provoque une augmentation significative de la surface foliaire. Pour le diamètre de pomme, une différence est observée pour les traitements $\mathrm{G} 2+\mathrm{FOV}+\mathrm{FMV}, \mathrm{G} 3+\mathrm{FOV} \mathrm{G} 3+\mathrm{FOV}+\mathrm{FMV}$ et PP par rapport au témoin. G1 est statistiquement identique à la dose la plus forte de tous les traitements $(\mathrm{G} 3+\mathrm{FOV}+\mathrm{FMV})$. En ce qui concerne la hauteur, les traitements $\mathrm{G} 2$, $\mathrm{G} 2+\mathrm{FOV}, \mathrm{G} 2+\mathrm{FOV}+\mathrm{FMV}$ et G3+FOV sont statistiquement différents comparés au témoin uniquement (Tableau 4).

\section{Effet du guano sur le rendement de laitue}

L'effet des traitements sur la croissance 
végétative de la laitue, est présenté dans le Tableau 5. Tant pour la biomasse fraîche que sèche, les traitements ont un effet significatif sur la production de la laitue. En prenant les doses du guano individuellement, il ressort que le rendement (frais) est inversemment proportionnel aux doses de guano appliquées , G3 (1,34 t/ha), G2 (2,41 t/ha) et G1 (3,58 t/ha). En combinant l'engrais chimique avec guano, le rendement oscille entre 0,62 et $1,07 \mathrm{t} / \mathrm{ha}$. L'apport conjoint d'engrais minéraux, fumier et guano a permis d'améliorer significativement le rendement pour les doses moyennes et fortes (G2 et G3) de guano par rapport au témoin. Ces résultats montrent également que le traitement G1+FOV est statistiquement identique à $\mathrm{G} 1, \mathrm{G} 2+\mathrm{FOV}$, $\mathrm{G} 2+\mathrm{FOV}+\mathrm{FMV}, \mathrm{G} 3+\mathrm{FOV}, \mathrm{G} 3+\mathrm{FOV}+\mathrm{FMV}$ et PP alors que le traitement FMV combiné aux autres doses de guano a eu un effet dépressif comparé aux parcelles traitées avec le guano uniquement.

L'analyse du rendement montre que, un apport du guano, avec ou sans engrais minéraux, améliore la production de la laitue. La même tendance est observée pour la biomasse sèche de laitue. Elle varie de 0,05 a $0,52 \mathrm{t} / \mathrm{ha}$. Une augmentation significative de la biomasse totale avec les traitements $\mathrm{G} 1+\mathrm{FOV}$, $\mathrm{G} 3+\mathrm{FOV}$ et $\mathrm{G} 3+\mathrm{FOV}+\mathrm{FMV}$ par rapport au témoin.

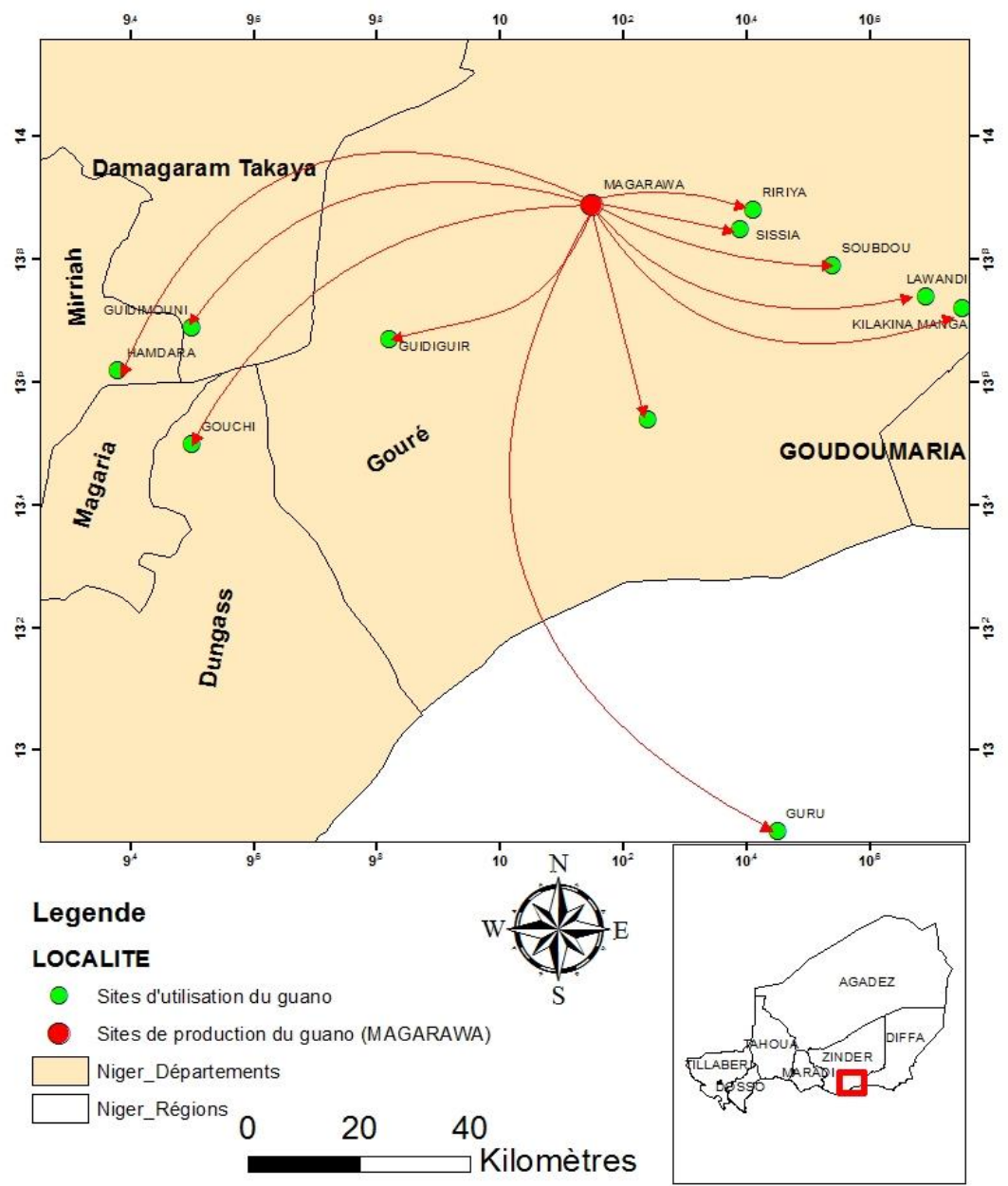

Figure 2 : Circuit de la commercialisation du guano de Magarawa. 
Tableau 1 : Prix de sac de $50 \mathrm{~kg}$ du guano à Magarawa de 2008 à 2012.

\begin{tabular}{lccc}
\hline Année & Prix d'achat (fcfa) & Prix de vente (fcfa) & Bénéfice (fcfa) \\
\hline 2008 & 200 & 750 & 450 \\
2009 & 250 & 1000 & 750 \\
2010 & 300 & 2500 & 2200 \\
2011 & 500 & 3000 & 2500 \\
2012 & 750 & 4000 & 3250 \\
\hline
\end{tabular}

Tableau 2 : Caractéristiques physico-chimiques du sol $(0-20 \mathrm{~cm})$ de la parcelle expérimentale.

\begin{tabular}{lc}
\hline Caractéristiques & Quantité \\
\hline $\mathrm{pH}$, eau (1:2.5) & $9,85 \pm 0,05$ \\
Carbone (C), \% & $3,9 \pm 0,36$ \\
$\mathrm{~N}$ total, \% & $0,2 \pm 0,04$ \\
$\mathrm{P}$ total, mg kg-1 & $546 \pm 3,06$ \\
$\mathrm{~K}, \mathrm{mg} \cdot \mathrm{kg}^{-1}$ & $10,4 \pm 0,21$ \\
$\mathrm{Ca}, \mathrm{mg} \cdot \mathrm{kg}^{-1}$ & $68540 \pm 9,04$ \\
$\mathrm{Mg}, \mathrm{mg} \cdot \mathrm{kg}^{-1}$ & $1186 \pm 7,09$ \\
$\mathrm{Na}, \mathrm{mg} \cdot \mathrm{kg}^{-1}$ & $3422 \pm 5,86$ \\
\hline Statut textural & Limoneux \\
\hline
\end{tabular}

Tableau 3 : Caractéristiques physico-chimiques du guano de Magarawa et du fumier de Balla.

\begin{tabular}{|c|c|c|c|c|c|c|c|c|c|}
\hline $\begin{array}{l}\text { Localité } \\
\text { d'origine }\end{array}$ & Paramètre & pH & $\begin{array}{l}\mathrm{CE} \\
\mathrm{mS} / \mathrm{cm}\end{array}$ & $\begin{array}{l}\mathbf{N} \\
\%\end{array}$ & $\begin{array}{l}P \\
\mathrm{mg} / \mathrm{kg}\end{array}$ & $\begin{array}{l}\mathrm{K} \\
\mathrm{mg} / \mathrm{kg}\end{array}$ & $\begin{array}{l}\mathrm{C} \\
\%\end{array}$ & $\begin{array}{l}\text { MO } \\
\%\end{array}$ & $\mathrm{C} / \mathrm{N}$ \\
\hline Magarawa & Guano & $9,12 \pm 0,1$ & $2,38 \pm 0,03$ & $9,8 \pm 0,01$ & $5090 \pm 10,1$ & $6571 \pm 3,2$ & $47 \pm 0,2$ & $80 \pm 0,1$ & $5 \pm 1,2$ \\
\hline Balla & Fumier & $8,02 \pm 0,2$ & $0,44 \pm 0,01$ & $1,1 \pm 0,04$ & $2531 \pm 4,02$ & $3491 \pm 5,2$ & $29 \pm 0,5$ & $50 \pm 0,3$ & $26 \pm 2,3$ \\
\hline
\end{tabular}


Tableau 4 : Effet du guano sur la croissance végétative de laitue.

\begin{tabular}{lccc}
\hline Traitement & Surface de la feuille $\left(\mathbf{c m}^{2}\right)$ & Diamètre de pomme $(\mathbf{c m})$ & Hauteur $(\mathbf{c m})$ \\
\hline T0 & $13,77 \mathrm{a}$ & $9,97 \mathrm{abc}$ & $3,5 \mathrm{ab}$ \\
FMV & $16,25 \mathrm{ab}$ & $10,88 \mathrm{abcd}$ & $4,42 \mathrm{abc}$ \\
FOV & $38,43 \mathrm{abcd}$ & $14,63 \mathrm{abcde}$ & $4 \mathrm{abc}$ \\
FOV+FMV & $29,79 \mathrm{abcd}$ & $11,55 \mathrm{abcd}$ & $4,83 \mathrm{abc}$ \\
G1 & $71,16 \mathrm{de}$ & $16,75 \mathrm{bcde}$ & $5,23 \mathrm{abcd}$ \\
G1+FMV & $13,64 \mathrm{a}$ & $8,94 \mathrm{a}$ & $3,83 \mathrm{abc}$ \\
G1+FOV & $64,30 \mathrm{cde}$ & $15,73 \mathrm{abcde}$ & $5,07 \mathrm{abc}$ \\
G1+FMV+FOV & $35,57 \mathrm{abcd}$ & $14,37 \mathrm{abcde}$ & $3,25 \mathrm{a}$ \\
G2 & $29,53 \mathrm{abcd}$ & $14,15 \mathrm{abcde}$ & $6,75 \mathrm{~cd}$ \\
G2+FMV & $14,35 \mathrm{ab}$ & $8,99 \mathrm{a}$ & $5,7 \mathrm{abcd}$ \\
G2+FOV & $63,53 \mathrm{cde}$ & $17,53 \mathrm{cde}$ & $6,83 \mathrm{~cd}$ \\
G2+FOV+FMV & $65,95 \mathrm{cde}$ & $19,4 \mathrm{e}$ & $8,25 \mathrm{~d}$ \\
G3 & $35,54 \mathrm{abcd}$ & $14,97 \mathrm{abcde}$ & $5,83 \mathrm{abcd}$ \\
G3+FMV & $26,09 \mathrm{abc}$ & $9,61 \mathrm{ab}$ & $4,5 \mathrm{abc}$ \\
G3+FOV & $85,28 \mathrm{e}$ & $23,87 \mathrm{e}$ & $6,92 \mathrm{~cd}$ \\
G3+FOV+FMV & $18,07 \mathrm{de}$ & $5,25 \mathrm{abcd}$ \\
PP & $56,41 \mathrm{bcde}$ & $20,82 \mathrm{e}$ & $6,40 \mathrm{bcd}$ \\
PPDS & $69,83 \mathrm{de}$ & 6,49 & 2,65 \\
F & 42,42 & $0,003 * *$ & $0,025 *$ \\
\hline Les & $0,008^{* *}$ & 5 &
\end{tabular}

Les moyennes affectées de la même lettre dans la même colonne ne sont pas significativement différentes au seuil de $5 \%$. PPDS: Plus Petite Différence Significative, *: significative; **: Très hautement significative.

Tableau 5 : Effet du guano sur le rendement.

\begin{tabular}{lll}
\hline Traitement & Rendement t/ha & Biomasse sèche $\mathbf{t} / \mathbf{h a}$ \\
\hline T0 & $0,43 \mathrm{a}$ & $0,05 \mathrm{a}$ \\
FMV & $0,57 \mathrm{a}$ & $0,08 \mathrm{ab}$ \\
FOV & $2,42 \mathrm{abcd}$ & $0,34 \mathrm{abcd}$ \\
FOV+FMV & $1,85 \mathrm{abcd}$ & $0,27 \mathrm{abcd}$ \\
G1 & $3,58 \mathrm{abcd}$ & $0,40 \mathrm{abcd}$ \\
G1+FMV & $0,90 \mathrm{a}$ & $0,11 \mathrm{ab}$ \\
G1+FOV & $4,58 \mathrm{~d}$ & $0,49 \mathrm{~cd}$ \\
G1+FMV+FOV & $2,00 \mathrm{abcd}$ & $0,26 \mathrm{abcd}$ \\
G2 & $2,41 \mathrm{abcd}$ & $0,22 \mathrm{abcd}$ \\
G2+FMV & $0,62 \mathrm{a}$ & $0,14 \mathrm{abc}$ \\
G2+FOV & $4,13 \mathrm{bcd}$ & $0,38 \mathrm{abcd}$ \\
G2+FOV+FMV & $4,19 \mathrm{bcd}$ & $0,41 \mathrm{abcd}$ \\
G3 & $1,34 \mathrm{abc}$ & $0,13 \mathrm{ab}$ \\
G3+FMV & $1,07 \mathrm{ab}$ & $0,13 \mathrm{ab}$ \\
G3+FOV & $4,27 \mathrm{~cd}$ & $0,44 \mathrm{bcd}$ \\
G3+FOV+FMV & $4,34 \mathrm{~cd}$ & $0,52 \mathrm{~d}$ \\
PP & $3,56 \mathrm{abcd}$ & $0,38 \mathrm{abcd}$ \\
PPDS 5\% & 2,69 & 0,30 \\
F & $0,01 *$ & $0,038^{*}$ \\
\hline
\end{tabular}

Les moyennes affectées de la même lettre dans la même colonne ne sont pas significativement différentes au seuil de 5\%. PPDS: Plus Petite Différence Significative; *: significative. 


\section{DISCUSSION}

Les qualités chimiques du guano contribuent à améliorer la production de la laitue verte par le biais de son action sur le sol. Ce travail a permis d'aboutir aux meilleures combinaisons de fertilisants pour une optimisation de la production de laitue verte. Il est donc techniquement facile et plus économiquement rentable pour un paysan d'utiliser la dose G1 afin d'optimiser sa production. Ceci pourrait être dû à la teneur élevée en azote du guano. Etant un élément constitutif de la chlorophylle, l'azote est un facteur déterminant dans la croissance et la détermination du rendement des plantes (Sikora et Szmidt, 2001; Douglas et al., 2003; Magnan, 2006). La différence entre la vitesse de minéralisation du guano beaucoup plus rapide comparée à celle des autres fertilisants expliquerait certainement l'efficacité en matière d'une meilleure production. En effet, le guano libère progressivement les minéraux, ce qui peut assurer leur disponibilité tout au long du développement de la plante. Les sources d'engrais organiques possédant un rapport $\mathrm{C} / \mathrm{N}$ inférieur à 20 contiennent une concentration élevée de nutriments (Chaves et al., 2007; Tognetti et al., 2008) et possèdent aussi des potentiels d'immobilisation de l'azote (De Neve et al., 2004). La valeur du pH $(9,12)$ et la teneur en azote $(9,8 \%)$ de ce guano sont supérieures à celles trouvées à Mbanza-Ngungu en République Démocratique du Congo respectivement 8,6 et 7,3 par Nzuki et al. (2011). Cette teneur en azote du guano de Magarawa $(9,8 \%)$ est nettement supérieur à celle trouvée au Bénin $(0,96 \%)$ par Biaou et al. (2017). Ceci montre que la qualité chimique du guano est en lien avec l'environnement de vie de la chauve-souris. En effet, la teneur des amendements organiques d'origine animale en éléments minéraux dépend de l'espèce animale, des aliments consommés et des modalités de stockage des déchets.

Oliveira et al. (2006) signalent que, l'apport des engrais organiques en faible quantité a donné un rendement de laitue très élevé, d'où la necessité de refléchir sur comment réduire la dose de guano et maintenir un bon niveau de production? Surtout que les résultats obtenus dans cette étude montrent des rendements statistiquement identiques pour G2+FOV, G2+FOV+FMV, G3+FOV; G3+FOV+FMV comparés à G1+FOV. Le fait d'introduire le fumier dans la fertilisation des sols de cuvette, contribue à ramener le $\mathrm{pH}$ du sol à un niveau légèrement plus acceptable en plus des compléments en fertilisants qu'il apporte. La matière organique améliore la fertilité du sol en agissant sur les propriétés physico-chimiques et biologiques du sol (Pietikainen et al., 2000). En outre, cette fumure se révèle souvent plus efficace que la fertilisation minérale (Soltner, 2003; Wang et al., 2012; Gunes et al., 2014). Au Burkina Faso, les études menées par Zougmoré et al. (2003) ont montré que l'application du fumier permet d'obtenir des rendements de 900 à $1600 \mathrm{~kg} / \mathrm{ha}$ de sorgho soit 20 à 39 fois le rendement obtenu sans aucun amendement. Selon Tchabi et al. (2012), la bouse de vache assure un bon développement de la culture de la laitue. Les résultats issus de cette étude valide le role que joue les amendements organiques dans l'amélioration de la production maraichère dans les systemes de production.

\section{Conclusion}

Cette étude a été menée dans le but de mesurer l'efficacité du guano avec les fertilisants couramment utilisés dans la production de la laitue verte dans le système oasien du Manga. L'optimisation de la production des plantes cultivées peut se faire par le biais des fertilisants, et le guano de chauves-souris (guano) en est un. Les résultats obtenus à l'issu de ce travail montrent que l'exploitation $\mathrm{du}$ guano est encore traditionnelle et les acteurs ne sont pas bien outillés tant dans l'exploitation que dans l'utilisation en culture maraichère. Cette situation fait que la population de Magarawa ne tire pas assez profit de l'exploitation de cette ressource d'une part et d'autre part, la dose traditionnelement utilisée par les producteurs de Balla ne donne pas les meilleures performances comparées aux autres combinaisons. L'expérimentation faite a montré aussi que le guano constitue un fertilisant riche en nutriments pouvant améliorer la production agricole. Les éléments chimiques qu'il contient sont plus elevés que ceux du fumier provenant du village de Balla. Cet engrais naturel permet d'obtenir avec la dose G1, un rendement presque 2 fois supérieur à celui du fumier vulgarisé (FOV) et 6 fois supérieur à celui de la fumure minérale vulgarisée (NPK + urée). Cela dénote que le guano peut avantageusement remplacer le fumier et l'engrais minéral. Toutefois, pour les producteurs, il est important de tenir compte de la vitesse de minéralisation du guano pour 
déterminer la période idéale d'apport des amendements organiques. Ainsi, du fait de leur caractéristique, le fumier pourrait être apporté en fumure de fond deux à trois semaines avant la mise en place des cultures, le guano étant employé en fumure d'entretien. $\mathrm{La}$ détermination des doses de la fertilisation en condition contrôlée pour les autres spéculations maraichères est sans aucun doute un vaste domaine à explorer. Le manque de références sur les besoins des cultures d'une part et la vitesse de minéralisation en fonction des types de sol d'autre part, pourraient servir de champs de recherche pour le futur. La connaissance de l'éthologie de la chauve-souris et du stock disponible de guano est un gage pour la durabilité de l'exploitation et de l'utilisation du guano. Pour une durabilité de cette exploitation du guano, il est impératif d'organiser les acteurs de la filière d'exploitation et d'utilisation en maraichage.

\section{CONFLIT D'INTERETS}

Les auteurs déclarent qu'ils n'ont aucun conflit d'intérêts.

\section{CONTRIBUTIONS DES AUTEURS}

MMM est l'auteur qui a conduit la recherche et a rédigé le manuscrit sous la supervision de ADT. KAH a participé à l'élaboration du protocole de recherche, la collecte et le traitement des données et des propositions pertinentes tout au long des travaux. MHM est celui qui a découvert l'utilisation du guano par les paysans des différentes cuvettes oasiennes du département de Gouré. Il a relu le document en donnant des pertinentes contributions.

\section{REFERENCES}

Biaou ODB, Saidou A, Bachabi F-X, Padonou GE, Balogoun I. 2017. Effet de l'apport de différents types d'engrais organiques sur la fertilité du sol et la production de la carotte (Daucus carota L.) sur sol ferralitique au sud Bénin. Int. J. Biol. Chem. Sci., 11(5): 2315-2326. DOI: https://dx.doi.org/10.4314/ijbcs.v11i5.29

Bouyoucos GJ. 1951. A recalibration of hydrometer for making mechanical analysis of soil. Agronomy Journal, 43: 434-438.

Chaves B, De Neve S, Piulats LM, Bocckx P, Van Cleemput O, Hofman G. 2007. Manipulation the $\mathrm{N}$ release from $\mathrm{N}$ - rich crop residues by using organic wastes on soils with different textures. Soil Use and Man., Univ Ghent, Dept Soil Management and Soil Care. Coupure Links 653, B-9000 Ghent, Belgium, 23: 212-219.

De Neve S, GaonaSae'z S, Chave B, Sleutel S, Hofman G. 2004. Manipulation N Mineralization from high $\mathrm{N}$ crop residues using on- and off farm organic materials. Soil Biology and Biochemistry, 36: 127134.

Dickman SR, Bray RH. 1940. Colorimetric determination of phosphate. Ind. Eng. Chem., Anal. Ed., 12: 665-668.

Douglas JT, Aitken MN, Smith CA. 2003. Effects of five non-agricultural organic wastes on soil composition and on the yield and nitrogen recovery on Italian ryegrass. Soil Use Man., 19: 135-138.

FAO, 2006. Sécurité alimentaire et développement agricole en Afrique Subsaharienne. Dossier pour l'accroissement des soutiens publics. p. 108.

Guengant JP, Banoin M. 2003. Dynamique des populations, disponibilités des terres et adaptation des régimes fonciers : le cas du Niger. FAO et Comité International de Coopération dans les Recherches Nationales en Démographie. Lututala M. (ed.). p. 144.

Gunes A, Inal A, Taskin MB, Sahin O, Kaya EC, Atakol A. 2014. Effect of phosphorus enriched biochar and poultry manure on growth and mineral composition of lettuce (Lactuca sativa L. cv.) grown in alkaline soil. Soil Use and Management, 30: 182-184.

Habou ZA, Boubacar MK, Adam T. 2016. Les systèmes de productions agricoles du Niger face au changement climatique : défis et perspectives. Int. J. Biol. Chem. Sci., 10(3): 1262-1272. DOI: http://dx.doi.org/10.4314/ijbcs.v10i3.28

Hillebrand WF, Lundell GKF, Bright HA, Hoffman J. 1953. Applied Inorganic Analysis ( $2^{\text {nd }}$ edn). John Wiled and Sons Inc.: New York, USA; 1034p.

Jackson ML. 1958. Soil Chemical Analysis. Prentice Hall.: New Jersey; 498 p.

Karen A. 2003. Nitrogen-PhosporusPotassium values of organic fertilizers. Oregen state Univesity extension services and educational programme: Oregen; 1-4.

Karimou AH. 2013. Effet du guano de chauvesouris sur la fertilité du sol et la 
production de la laitue verte (Lactuca sativa) dans la cuvette de Balla, Mémoire de master 2 Gestion Intégrée de la fertilité des sols, Institut du développement rural, Université Polytechnique de BoboDioulasso, Bobo-Dioulasso, p. 98.

Magnan J. 2006. Epandage post récolte des engrais organiques et risques environnementaux reliés aux pertes d'azote. Ordre des Agronomes du Québèc., p.75.

Malam Boukar AK, Tıdjanı AD, Yamba B, Lebailly P. 2016. Performance et circuit de commercialisation des principaux produits agricoles des cuvettes oasiennes du département de Gouré (Niger). Int. J. Biol. Chem. Sci., 10(5): 2202-2214. DOI: http://dx.doi.org/10.4314/ijbcs.v10i5.21

Mentler A, Partaj T, Strauss P, Soumah M Blum WE. 2002. Effect of locally available organic manure on maize yield in Guinea. West Africa.17 th WCSS, 1421. August, Thailand. Sympos. 13. Pap. 2029: $1-8$

Mulambuila MN, Kamambo RM, Jadica CT, Tshibamba JM, Mukanya MB. 2015. Étude comparative de quelques fertilisants (Bat-guano et DAP) sur le rendement du niébé (Vigna unguiculata, L. Walp.) dans la région de Gandajika (RDC). J. Appl. Biosci., 92: 8651-8658. DOI: http://dx.doi.org/10.4314/jab.v92i1.9

Ndiaye M, Madai A, Thiers R. 2013. Implementing the MAF in Niger: Opportunities and challenges in accelerating MDG 1. UNDP Working Paper. UNDP Publishing. http://hdl.handle.net/1854/LU-5720228

Nzuki BF, Kinkwono EK, Sekle BG. 2011. Utilisation du guano comme substitut du Di-ammonium Phosphate (DAP) dans la fertilisation du soja et de la tomate en République Démocratique du Congo. Tropicultura, 29(2) : 114-120.

Oliveira NG, De Polli H, Almeida DL, Guerra JGM. 2006. No tillage system of lettuce fertilized with poultry litter on living roofs of grass and legume. Hortic. Bras., p. 24.

Pietikainen J, Kiikkilä O, Fritze H. 2000. Charcoal as a habitat for microbes and its effect on the microbial community of the underlying humus. Oikos, 89: 231-242.

Pratt PF. 1965. Methods of soil analysis. Part II. Chemical and Microbiological Properties, Black CA (ed). American Society of Agronomy: Madison; 7711572.

RECA. 2015. Fiche technique pour la culture de la laitue / salade. Version (1), p. 2.

Sikora LJ, Szmidt AK. 2001. Nitrogen sources, mineralization rates, and nitrogen nutrition benefits to plants from composts. In Compost Utilization in Horticultural Cropping Systems, Stoffella PJ, Kahn BA (eds). Lewis Publishers: New York, USA; 287-305.

Soltner D. 2003. Les bases de la production végétale, le sol et son amélioration, Tome 1. 23è Ed. Poitiers, France, p. 472.

Sridhar KR, Ashwini KM, Seena S, Sreepada KS. 2006. Manure qualities of guano of insectivorous cave bat Hipposideros speoris. Tropical and Subtropical Agroecosystems, 6 (2): 103-110.

Tognetti C, Mazzarino MJ, Laos F. 2008. Compost of municipal organic waste: effects of different management pratrices on degrability and nutrient release capacity. Soil Biology Biotechnology, 49: 2290-2296.

Tchabi VI, Azokli D, Biaou GD. 2012. Effet de différentes doses de bouse de vache sur le rendement de la laitue (Lactuca sativa $\mathrm{L}$.) à Tchatchou au Bénin. Int. J. Biol. Chem. Sci., 6(6): 5078-5084. DOI: http://dx.doi.org/10.4314/ijbcs.v6i6.26

Walkley A, Black IA. 1934. An examination of the Degtjareff method for determining soil organic matter and a proposed modification of the chromic acid titration method. Soil Sci., 37: 29-38.

Wang T, Arbestan MC, Hedley M, Bishop P. 2012. Predicting phosphorus bioavailability from high-ash biochars. Plant and Soil, 357: 173-187.

Zougmoré R, Zida Z. Kambou NF. 2003. Role of nutrient amendments in the success of half-moon soil and water conservation practice in semi-arid Burkina Faso. Soil and Tillage Research, 71: 143-149. 